\title{
Effect of Long-Term Exposure to Constant Dim Light on the Circadian System of Rats
}

\author{
Chiaki Fukuhara Jacopo Aguzzi Nicole Bullock Gianluca Tosini
}

Neuroscience Institute and NSF Center for Behavioral Neuroscience, Morehouse School of Medicine, Atlanta, Ga., USA

\section{Key Words}

Suprachiasmatic nucleus $\cdot$ Retina $\cdot$ Pineal gland . Arylalkylamine $\mathrm{N}$-acetyltransferase $\cdot$ Melatonin .

Desynchronization, internal $\cdot$ Period 1 gene

\begin{abstract}
The newly discovered multi-oscillatory nature of the mammalian circadian clock system and the cloning of the genes involved in the molecular mechanism that generates circadian rhythmicity have opened new approaches for understanding how mammals are temporally organized and how the mammalian circadian system reacts to the lack of normal synchronization cues. In the present study we investigated the effects of longterm exposure to constant red dim light on the pattern of the expression of Period 1 in the suprachiasmatic nuclei of the hypothalamus and of Arylalkylamine $\mathrm{N}$-acetyltransferase (Aa-nat) in the retina and pineal gland. Our data demonstrate that Period 1 mRNA expression in the suprachiasmatic nuclei of the hypothalamus was not affected by exposure to constant red dim light for 60 days, whereas Aa-nat mRNA expression in the retina and in the pineal gland was significantly affected, since in some animals (20-30\%) Aa-nat mRNA levels were found
\end{abstract}

to be higher during the subjective day. A circadian rhythm of serum melatonin and locomotor activity was present in all the animals tested. In 4 animals serum melatonin levels were high during the subjective day. Our data suggest that long-term exposure to constant red dim light may induce desynchronization between the circadian rhythm of locomotor activity and serum melatonin levels.

Copyright $\odot 2005$ S. Karger AG, Basel

\section{Introduction}

Daily rhythmicity is a ubiquitous feature of living systems. Generally, these rhythms are not just a passive consequence of cyclic fluctuations in the environment, but rather they originate within the organisms: they are generated and controlled by endogenous physiological oscillators. The fundamental adaptive function of an endogenously programmed rhythmicity is to provide an optimal and anticipatory temporal organization of physiological processes and behavior in relation to the environment [1]. Synchronization of endogenous circadian rhythms to the 24-hour environment occurs through daily adjustment of the clock by external time cues or zeitgebers. The most

\section{KARGER}

Fax + 41613061234 E-Mail karger@karger.ch www. karger.com
(C) 2005 S. Karger AG, Base

1424-862X/05/0143-0117\$22.00/0

Accessible online at:

www. karger.com/nsg
Gianluca Tosini, $\mathrm{PhD}$

Neuroscience Institute, Morehouse School of Medicine

720 Westview Dr. SW

Atlanta, GA 30310-1495 (USA)

Tel. +1 404756 5214, Fax +1 404752 1041, E-Mail tosinig@msm.edu 
important and reliable cue for synchronization is the daily light-dark cycle [2].

The newly discovered multi-oscillatory nature of the mammalian circadian clock system [3] and the cloning of the genes involved in the molecular mechanisms that generate circadian rhythmicity [4] have opened new approaches for understanding how mammals are temporally organized, and how the mammalian circadian system reacts to the lack of normal synchronization cues.

The primary goal of the present study was to determine how the circadian rhythms in the suprachiasmatic nuclei (SCN) of the hypothalamus, retina and pineal are affected by long-term exposure to constant red $\operatorname{dim} \operatorname{light}\left(\mathrm{LL}_{\mathrm{dim}}\right)$. Previous work has demonstrated that a clear phase relationship exists among the pattern of expression (in the SCN and elsewhere) of the clock genes belonging to the Period family and the locomotor activity rhythm [5-8]. In addition, other studies have demonstrated that the pattern of expression of Arylalkylamine $N$-acetyltransferase (Aa-nat, i.e., the gene that is responsible for the synthesis of the key forming enzyme in melatonin synthesis) in the retina and the pineal gland is under circadian control, and its expression shows a fixed and dependable phase relationship with the circadian rhythm of locomotor activity [for review see 5, 9, 10]. This experimental evidence suggested that the pattern of expression of clock genes (e.g. Period 1 [Per 1]) and clock-controlled genes (e.g., Aa-nat) could be used as reliable markers of the circadian phase in different organs, and thus could be utilized to investigate how exposures to constant conditions affect the circadian system.

In the present study we investigated how circadian rhythms in the SCN (by assessing locomotor activity and Perl) mRNA levels, in the retina and in the pineal (by measuring Aa-nat mRNA and serum melatonin levels) are affected by prolonged exposure to constant $\mathrm{LL}_{\mathrm{dim}}$.

\section{Materials and Methods}

\section{Experimental Animals}

Three groups ( $\mathrm{n}=36$ in each group) of male Wistar (Charles River Laboratory) rats, 8 weeks old at the start of experiment, were used in this study. During the experiment, the rats had free access to food and water.

\section{Monitoring of Wheel-Running Activity}

Animals were housed in cages equipped with a running wheel and a magnetic sensor system to detect wheel rotation (Mini-Mitter Co., Sunriver, Oreg., USA). Wheel-running data were recorded in 5-min bins saved on a PC using specific software (Tau, Mini-Mitter Co.) and were analyzed with specific software (VitalView, Mini-Mitter
Co.) to determine rhythmicity and the free-running period. Period was estimated by $\chi^{2}$ periodgram analysis.

\section{Housing and Tissue Collection and Assessment of the Circadian}

Phase

Animals were housed in a temperature controlled $\left(23-24^{\circ} \mathrm{C}\right)$ ventilated lightproof room. The animals were kept in a light:dark (LD) cycle or in constant dim illumination ( $\left.\mathrm{LL}_{\mathrm{dim}}, 1 \mu \mathrm{W} / \mathrm{cm}^{2}, 5 \mathrm{Lux}\right)$. In LD the onset of light was defined as zeitgeber time (ZT) 0 , and the onset of darkness as ZT12. In $\mathrm{LL}_{\text {dim }}$ light was provided by a special fluorescent fixture (Litho light No. 2, lower wavelength cutoff at $640 \mathrm{~nm}$ ). In all the experiments when animals were sacrificed during the dark phase, the procedure was carried out in $\mathrm{LL}_{\mathrm{dim}}$. Retinas and pineal glands were removed under $\mathrm{LL}_{\mathrm{dim}}$ (except for the sampling during the light phase).

After 0 (LD), 5, and 60 days of exposure to $\mathrm{LL}_{\mathrm{dim}}$, animals (6 individuals for each time point) were sacrificed at six different circadian times (CTs): 3 during the subjective day (CT4, CT8 and CT12) and 3 during the subjective night (CT16, CT20 and CT24), and the $\mathrm{SCN}$, retinas and pineal glands were collected and immediately frozen on dry ice. In order to ensure that each animal was sacrificed at the exact circadian time, the following procedure was adopted. For each animal, the time of sacrifice was determined based upon each animal's locomotor activity rhythm. CT12 was defined as the time at which an animal began its daily bout of wheel-running activity. Wheel-running records were plotted, and a line was drawn through activity onsets in order to calculate CT12 on the day of sacrifice. All other circadian times were calculated relative to CT12.

\section{Semiquantitative in situ Hybridization}

Blocks of brain containing the SCN region were obtained using razor blades, immediately frozen using dry ice, and kept at $-80^{\circ} \mathrm{C}$ until sectioning. Pieces of brain were then sliced into $20-\mu \mathrm{m}$-thick sections. For hybridization at least 5 sections from the medial part of the SCN were used for each animal. A 982-bp fragment of rat Per 1 cDNA cloned in the pGEM-T easy vector (Promega) was linearized by $\mathrm{SalI}$ or $\mathrm{SacII}$ for antisense or sense probe. $\left[{ }^{35} \mathrm{~S}\right]-\mathrm{UTP}-l a b e l e d$ antisense and sense probes were obtained by in vitro transcription using T7 or SP6 RNA polymerase. Hybridization was performed overnight at $55^{\circ} \mathrm{C}$. Following $30 \mathrm{~min}$ of RNase A treatment at $37^{\circ} \mathrm{C}$, sections were washed, dehydrated by ethanol, and air-dried. The slides were then exposed to Kodak MR film with ${ }^{14} \mathrm{C}$ standards (Amersham Life Science) for 4-6 days at room temperature.

Each autoradiography was subjected to quantification. Image of autoradiography films was scanned using a high-resolution scanner (U-MAX, PowerLook III) and optical density (OD) values for sense and antisense probes were determined using $\mathrm{NIH}$ image (version 1.62) on a Macintosh (G 3) computer. OD values for the sense probe were subtracted from OD values of the anti-sense probe. At least 5 sections from 1 animal were used and averaged OD values were represented as one animal's OD value. All the slides were stained using toluidine blue for evaluation of the SCN region and tissue conditions [for further details see 11,12 ].

\section{Real-Time Quantitative RT-PCR}

Total RNA from the retina and pineal gland was extracted using Trizol reagent (Gibco, BRL) following sonication. DNA was degraded by DNase I (Boehringer Mannheim). One set of sample (ZT or CT $4,8,12,16,20,24)$ was subjected to all the procedures (RNA extraction, DNase I treatment, cDNA synthesis, and PCR) at the 
same time. Real-time quantitative RT-PCR was carried out using an $i$ Cycler (Biorad). Standards that contained a known amount of Aa-nat or Gapdh plasmid DNA were used to generate standard curves during each run. We used the following primers: Gapdh (5'-AGACAGCCGCATCTTCTTGT-3', 5'-TGATGGCAACAATGTCCACT-3'), and Aa-nat (5'-TCACTGAGCTGCAATGTTCC-3', $5^{\prime}$-GATGTCAGCCTGGCCTAGAG-3'). Forty cycles of amplification were carried out following $15 \mathrm{~s}$ of denaturation at $95^{\circ} \mathrm{C}$. Once the temperature reached $95^{\circ} \mathrm{C}$, it was decreased to $60^{\circ} \mathrm{C}$, maintained for $45 \mathrm{~s}$, and raised to $72^{\circ} \mathrm{C}$ for $30 \mathrm{~s}$. Fluorescence (Sybr Green) was measured at melting temperature after each cycle, rather than at the end of 40 cycles of amplification, which allows comparison of fluorescence intensities among samples while their increase is within linear regression. Melting temperatures used were $86^{\circ} \mathrm{C}$ for Gapdh and $89^{\circ} \mathrm{C}$ for Aa-nat.

After RT-PCR, PCR products were run on the agarose gel to verify the quality of products. The amount of RNA was estimated based on the standard by computer software (The LightCycler Software Version 3). Aa-nat mRNA levels were normalized by Gapdh [13].

\section{Blood Sampling and Radioimmunoassay}

Thirteen rats were exposed to constant $\mathrm{LL}_{\mathrm{dim}}$ for 60 days, then blood samples $(80-100 \mu M)$ were collected every $4 \mathrm{~h}$ from the tail artery in heparinized tubes, stored overnight at $4{ }^{\circ} \mathrm{C}$, and then centrifuged at 1,500 rpm. For each animal, the time of sampling was determined based upon each animal's locomotor activity rhythm. CT12 was defined as the time at which an animal began its daily bout of wheel-running activity. Wheel-running records were plotted, and a line was drawn through activity onsets in order to calculate CT12 on the day of sacrifice. All other circadian times were calculated relative to CT12. Control samples were obtained from 2 rats kept in LD for 60 days. After that the serum was stored at $-80^{\circ} \mathrm{C}$ until the samples were processed for radioimmunoassay. Melatonin was extracted from the serum $(50 \mu M)$ using chloroform and then melatonin levels were measured using a commercially available kit (Alpco Diagnostics). The sensitivity of the assay was $0.2 \mathrm{pg} / \mathrm{ml}$. Intra-assay variability was $7 \%$ and the inter-assay variability was $11 \%$.

\section{Results}

\section{Wheel-Running Activity}

In all animals tested, clear circadian rhythms in locomotor activity were observed. As expected, the free-running period was longer than $24 \mathrm{~h}$ (for all the animals tested the mean \pm SEM was $24.9 \pm 0.12$ ). There was no noticeable difference in the locomotor activity pattern (i.e., in the free-running period or in the duration of the activity) during the duration of the whole experiment (fig. 1).

\section{Per1 Expression in the $S C N$}

Using in situ hybridization we investigated the pattern of Per 1 gene expression in the SCN. Per 1 expression in the SCN showed robust rhythmicity in animals kept in LD cycles (fig. 2; Kruskal-Wallis, $\mathrm{p}<0.05$ ), the rhythmicity persisted in animals exposed to $\mathrm{LL}_{\mathrm{dim}}$ for 5 days (fig. 2;
Kruskal-Wallis, $\mathrm{p}<0.05)$, and in animals that have been exposed to $\mathrm{LL}_{\mathrm{dim}}$ for 60 days (fig. 2; Kruskal-Wallis, $\mathrm{p}<$ 0.05). In all three conditions the Per 1 expression peaked during the middle of the day (subjective day for the animals held in constant conditions) around CT4-8. The only noticeable differences between LD and 60 days in $\mathrm{LL}_{\mathrm{dim}}$ were a small reduction in the amplitude of the rhythm and an increase in the overall variability (fig. 2).

\section{Aa-nat Expression in the Retina}

In LD conditions, Aa-nat mRNA levels showed marked day-night variations (fig. 3; Kruskal-Wallis, $\mathrm{p}<$ 0.05). The retinal Aa-nat mRNA rhythm persisted after 5 days of $\mathrm{LL}_{\mathrm{dim}}$ conditions (fig. 3; Kruskal-Wallis, $\mathrm{p}<0.05$ ). After 60 days in $\mathrm{LL}_{\mathrm{dim}}$ Aa-nat mRNA levels showed an increase in the overall variability at each $\mathrm{CT}$ analyzed (fig. 3). In some animals Aa-nat mRNA levels were found to be high during the subjective day (CT 4-12) or low during the subjective night (CT16-24).

\section{Aa-nat Expression in the Pineal}

In LD conditions, pineal Aa-nat mRNA levels showed a robust day-night variation (fig. 4; Kruskal-Wallis, $\mathrm{p}<$ 0.05). Aa-nat mRNA levels were very low during the day and higher at night. Pineal Aa-nat mRNA rhythm persisted after 5 days of $\mathrm{LL}_{\mathrm{dim}}$ conditions (fig. 4; KruskalWallis, $\mathrm{p}<0.05$ ); Aa-nat signals were observed throughout the day with the highest level at CT16-20 and the lowest at CT12. After 60 days in $\mathrm{LL}_{\text {dim }}$ (fig. 4), in a few animals $A a$-nat mRNA levels were high during the subjective day (CT4-12) or low during the subjective night (CT1624; fig. 4).

\section{Serum Melatonin}

In figure 5 are shown the serum melatonin profiles over a 24-hour period for 2 controls (i.e., animals exposed to LD) and 13 experimental animals (i.e., animals exposed to $L L_{\text {dim }}$ for 60 days). All the animals showed significant circadian melatonin rhythms $(\mathrm{p}<0.01)$. In controls melatonin levels were high during the night and low during the day (fig. 5A, B), the same profile was also observed in the majority $(n=9)$ of the experimental animals (fig. 5C-E, G-I, K, M, N). In 4 animals serum melatonin levels were higher during the subjective day (CT4-12) than during the subjective night (fig. $5 \mathrm{~F}, \mathrm{~J}, \mathrm{~L}, \mathrm{O}$ ). 

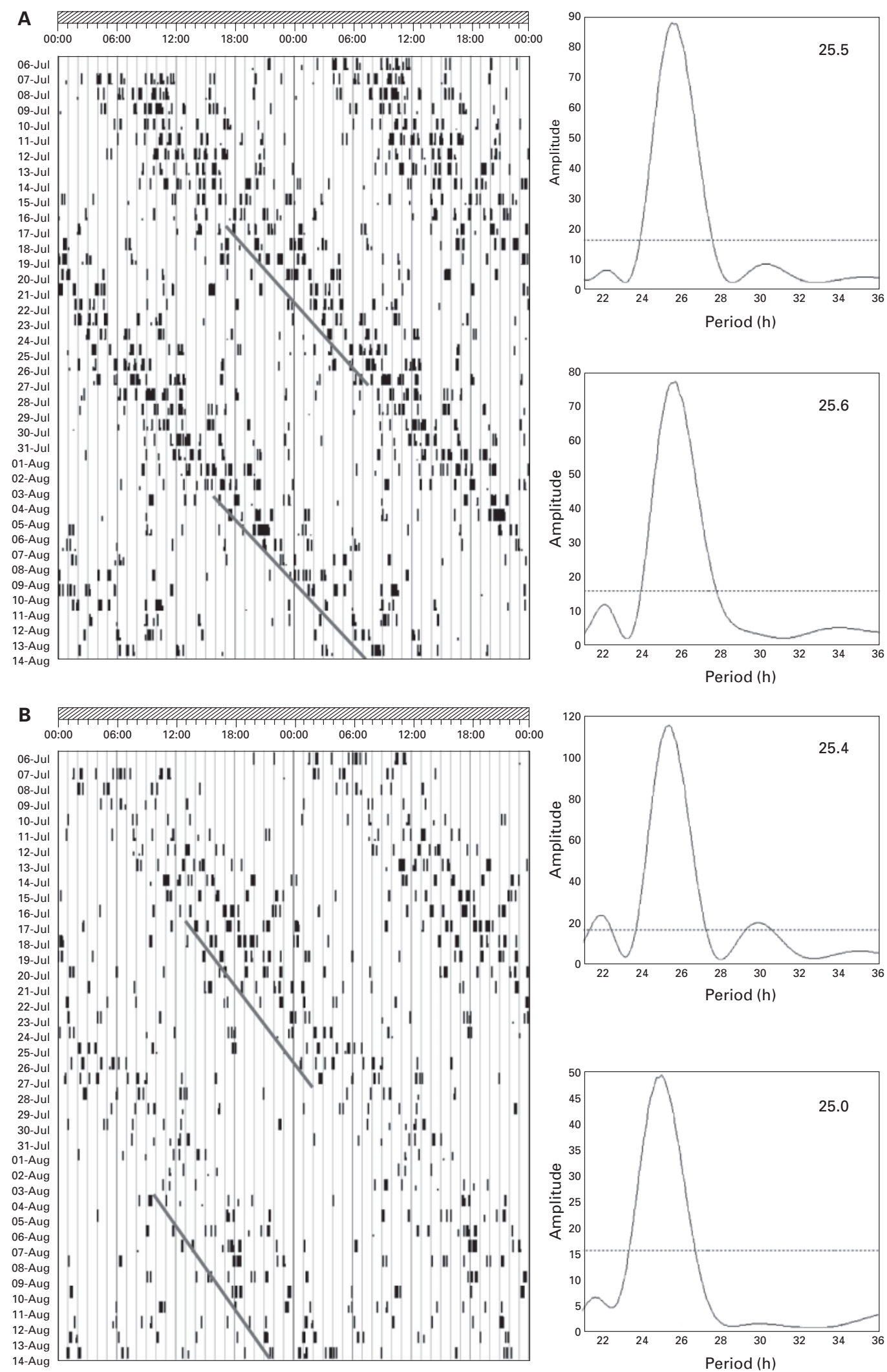


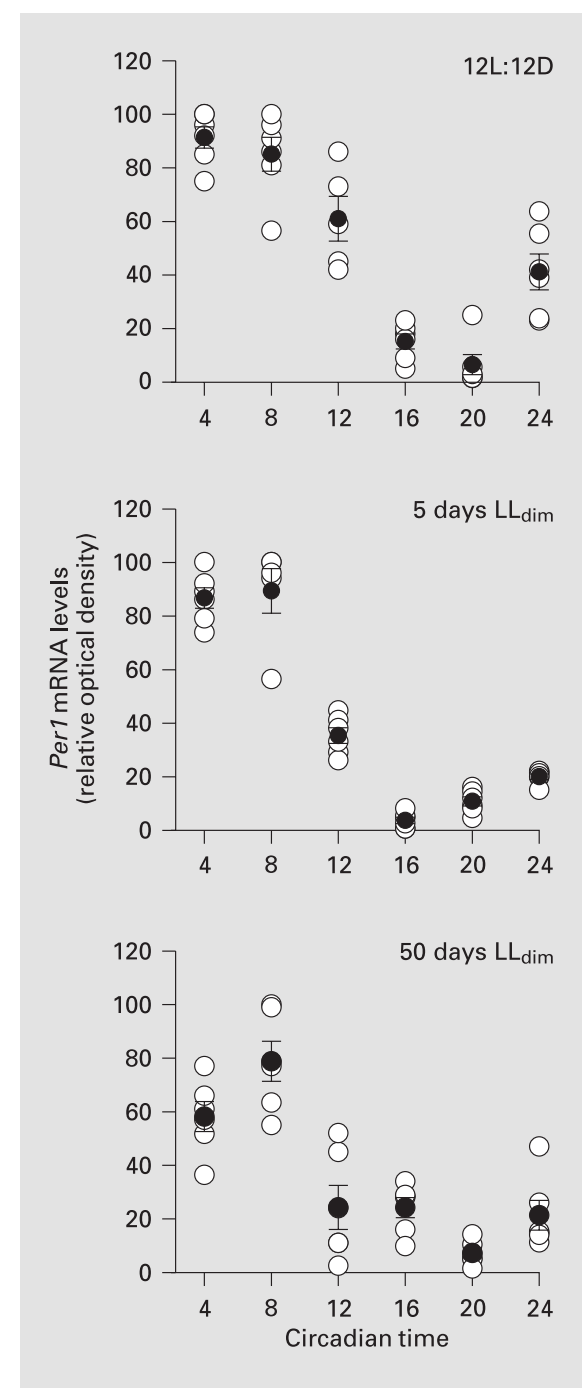

Fig. 2. Circadian variation of Perl mRNA levels in the SCN measured using semiquantitative in situ hybridization. 12L:12D; after 5 days in $L L_{\text {dim }}$ and after 60 days in $L_{\text {dim }}$. The white circles represent individual values; the black circles represent the mean ( \pm SEM) values $(n=6$ for each time point $)$.

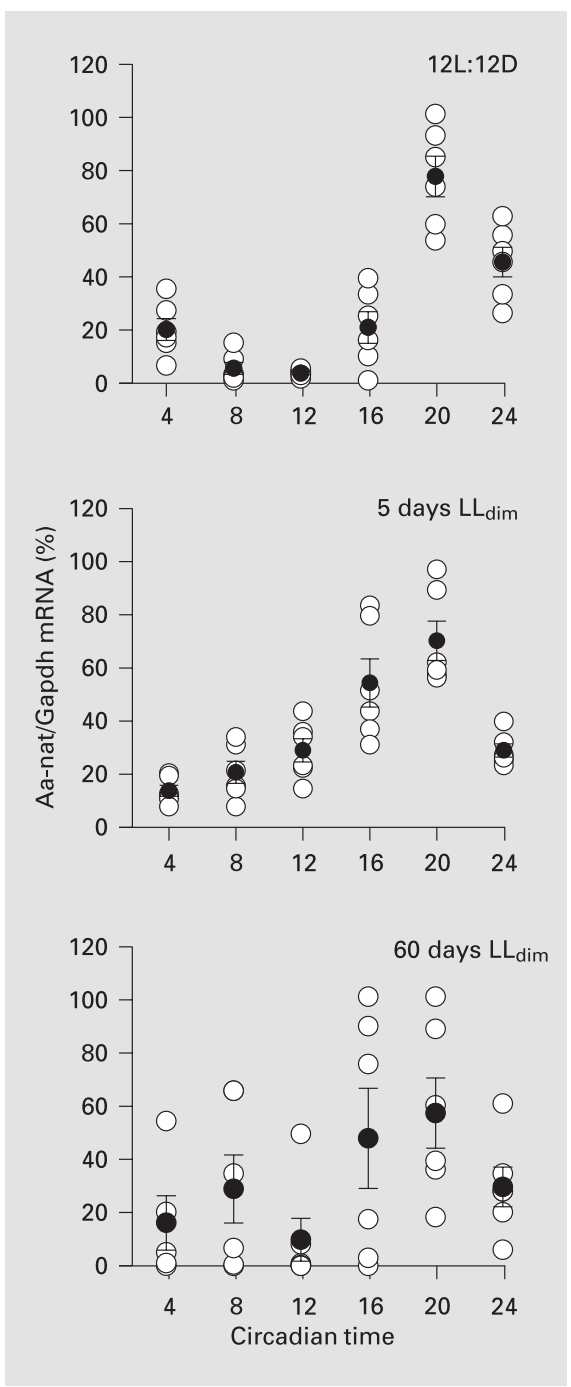

Fig. 3. Circadian variation of Aa-nat mRNA levels in the retina. 12L:12D; after 5 days in $\mathrm{LL}_{\text {dim }}$ and after 60 days in $\mathrm{LL}_{\text {dim }}$. The white circles represent individual values, while the black circles represent the mean $( \pm$ SEM) values ( $n=6$ for each time point).
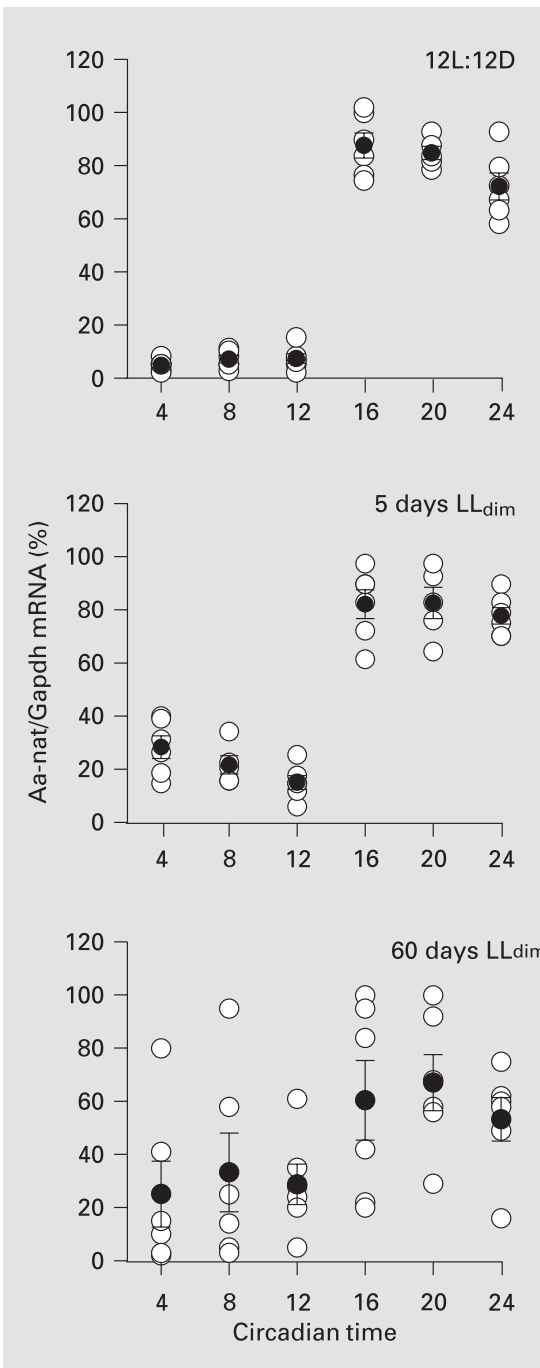

Fig. 4. Circadian variation of $A a$-nat mRNA levels in the pineal. 12L:12D; after 5 days in $\mathrm{LL}_{\mathrm{dim}}$ and after 60 days in $\mathrm{LL}_{\mathrm{dim}}$. The white circles represent individual values, while the black circles represent the mean $( \pm$ SEM) values ( $\mathrm{n}=6$ for each time point).

Fig. 1. Representative double plotted activity records (from day 20 to day 60) for 2 rats exposed to $L_{L_{\text {dim }}}$ for 60 days. In these animals, as in all the others, a clear circadian rhythm in locomotor activity was present for entire duration of the experiment. Small changes in the free-running period were observed (see periodogram analysis next to each actogram; The lines indicate the onset of activity and the interval of time in which the periodgram was calculated). The serum melatonin levels for these animals are shown in figure $5 \mathrm{D}(\mathbf{A})$ and $5 \mathrm{~J}(\mathbf{B})$. 

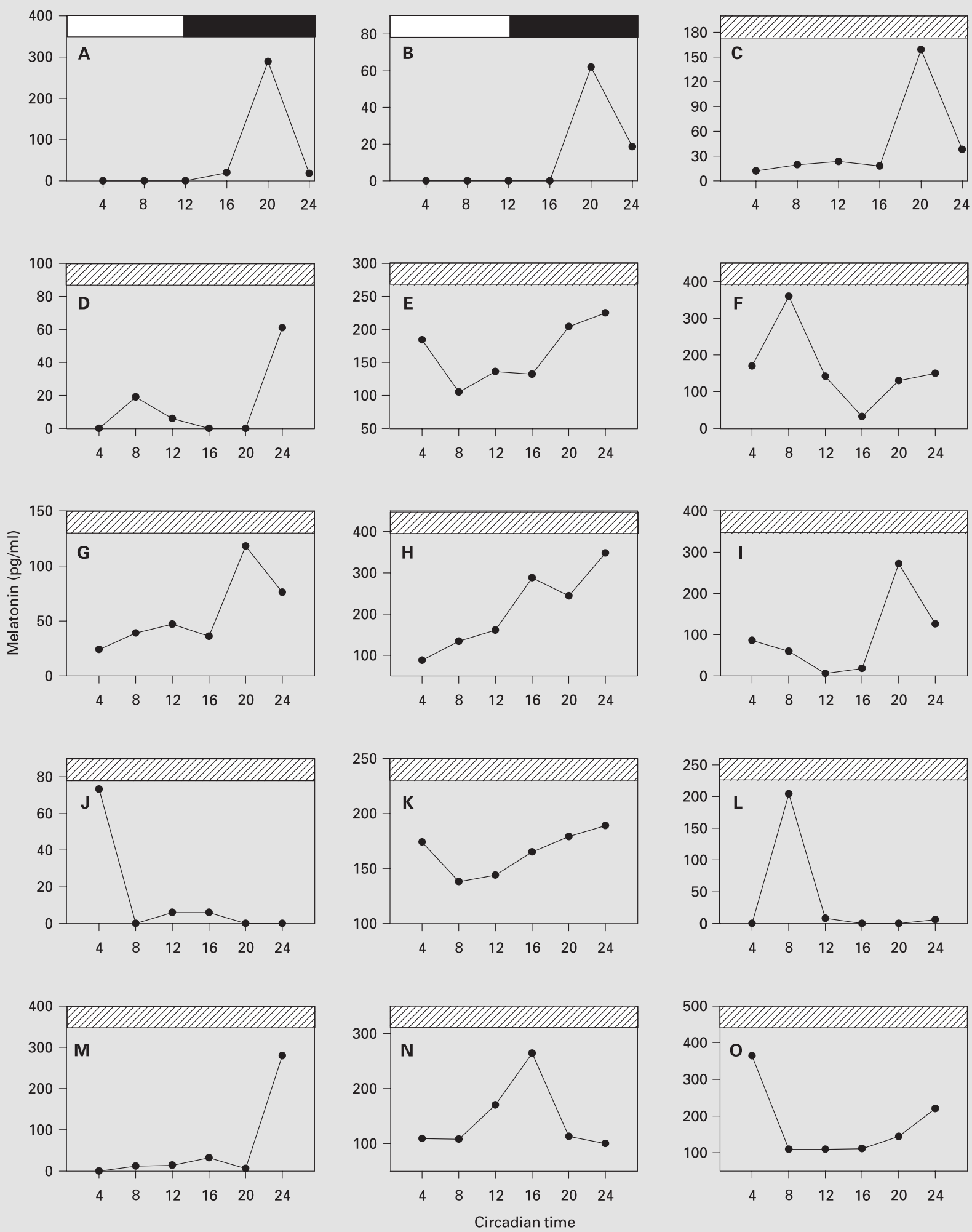

Fig. 5. Serum melatonin profile in animals exposed to $\operatorname{LD}(\mathbf{A}, \mathbf{B})$ and after 60 days of exposure to $\operatorname{LL}_{\text {dim }}(\mathbf{C}-\mathbf{O})$. In the control animals and in most of the experimental animals serum melatonin levels were high during the subjective night (ZT16-24), whereas in 4 animals serum melatonin levels were high during the subjective day (F, $\mathbf{J}, \mathbf{L}$ and $\mathbf{O})$. 


\section{Discussion}

At the core of the system that controls and regulates circadian rhythms in mammals there are three structures, i.e., the retina, the pineal gland and the $\mathrm{SCN}$, that together with interconnection form a central circadian axis [14]. The present work demonstrates that long-term exposure to $L L_{\text {dim }}$ has profound effects on this axis, since it affects the circadian transcription of Aa-nat mRNA in the retina and pineal gland. However it is important to note that all the animals tested showed a robust circadian rhythm in the levels of circulating melatonin and in the locomotor activity rhythm. Interestingly in a few animals $(\mathrm{n}=4)$ melatonin levels peaked during the daytime (CT4-8). Such a result suggests that exposure to $\mathrm{LL}_{\mathrm{dim}}$ may induce desynchronization between the circadian rhythm of locomotor activity and the circadian rhythm of serum melatonin.

Our data are consistent with previous investigations that have demonstrated that Per 1 mRNA transcription in the SCN is rhythmic in LD and this rhythm is maintained when the animals are released in constant conditions [68]. The data presented here expand these previous results by demonstrating that circadian expression in Per 1 mRNA in the SCN is maintained in animals that have been exposed to $L L_{\text {dim }}$ for 60 days (fig. 2). Such a finding suggests that the expression of this gene in the SCN is not significantly affected by long-term exposure to $L L_{d i m}$. This is not surprising, since a previous study has shown that Per 1 expression in cultured SCN can be maintained for at least 32 cycles [15].

Previous investigations have also shown that Aa-nat mRNA expression in the pineal gland and retina is rhythmic in $\mathrm{LD}$, and after few days in a constant lighting condition $[5,10]$. Our data agree well with these previous reports. Different from what we have observed in the SCN, Aa-nat mRNA in the retina and pineal gland was significantly affected by long-term exposure to $L_{L_{\text {dim }}}$, since in some animals $A a-n a t$ mRNA levels were found to be high in the subjective day and low in the subjective night (fig. 3, 4).

An important question that arises from these data is the physiological significance of the results obtained in this study with respect to the relationship between locomotor activity and Aa-nat mRNA. Several studies have shown that in the rat Aa-nat mRNA is the rate-limiting enzyme in the melatonin biosynthetic pathway and that changes in the transcription rate will induce changes in the protein levels and activity, and thus in the levels of melatonin [for review see 9]. Therefore, it is likely that the observed alteration of Aa-nat mRNA levels may induce in significant changes in the circadian profile of the circulating melatonin.

To further support our inference, we investigated the circadian rhythms of serum melatonin in rats exposed to $\mathrm{LL}_{\mathrm{dim}}$ for 60 days. As shown in figure 5C-O melatonin was detected in the serum and showed a robust circadian rhythm in all the animals tested, thus demonstrating that exposure to $\mathrm{LL}_{\mathrm{dim}}$ does not abolish melatonin synthesis. The levels of circulating serum melatonin were similar to those observed in previous studies [16] and no significant differences were observed between control and experimental animals (fig. 5). In most of the animals the phase of the melatonin rhythm was similar to that observed in the control animals. In 4 animals the melatonin rhythm was uncoupled from the locomotor activity rhythm since it peaked during the subjective day (fig. 5E, F, J, O), thus confirming the results obtained with pineal Aa-nat mRNA (fig. 4).

Such a result further indicates that in some animals long-term exposure to $\mathrm{LL}_{\mathrm{dim}}$ may uncouple the circadian of locomotor activity from the circadian rhythm of Aa-nat mRNA and serum melatonin levels. Furthermore, our data suggest that the uncoupling of the melatonin circadian from the locomotor activity rhythm occurs without affecting the overall pattern of activity and Perl expression in the SCN.

Alteration in the rhythm of melatonin may produce important effects on the organism. For example, altering melatonin levels during the daytime has a significant effect on sleep, mood, and performance [17], and motor performance [18].

Internal desynchronization among circadian rhythms has been reported only by a few studies. For example, spontaneous internal desynchronization between the body temperature rhythms and locomotor activity rhythms was observed in reptiles [19] and in the squirrel monkey [20]. In the owl monkey internal desynchronization between circadian activity and the feeding patterns has been also observed [21]. A recent study has reported that exposure to dim illumination may uncouple several (e.g., sleep, body temperature, locomotor activity and drinking) circadian rhythms in the rat [22]. Internal desynchronization has also been reported in humans [23, 24 ], and it is believed to be the cause of several pathologies $[25,26]$. The data presented demonstrate that desynchronization may occur between the locomotor activity rhythm and the molecular mechanisms controlling melatonin synthesis. 
Since it is well established that the mammalian retina contains an autonomous circadian clock that directly controls Aa-nat mRNA [27] and melatonin synthesis [28], it is likely that the desynchronization observed in some animals between retinal Aa-nat mRNA and locomotor activity rhythm is the consequence of the uncoupling between the retinal clock and the SCN clock. On the other hand an explanation of the results obtained in the pineal gland is more complicated. Aa-nat mRNA in the rat pineal gland is directly regulated by the $\mathrm{SCN}$ via a multisynaptic pathway [29]. Although recent experimental evidence has suggested that the mammalian pineal may also contain a circadian clock [30], it is unlikely that Aa-nat mRNA is regulated by this endogenous clock since the mechanism by which the circadian clock directly controls the Aa-nat gene is not functional in the mammalian pineal gland [27]. Therefore, we believe that the mechanisms responsible for the desynchronization of Aa-nat mRNA in the pineal gland may reside within the SCN and/or in the pathway connecting the SCN and the pineal gland. This belief is based on the fact that the SCN is composed of different populations of circadian oscillators that constitute regional pacemaker-controlling specific circadian outputs. For example, the circadian rhythm of arginine vasopressin and vasoactive intestinal polypeptide release in the rat $\mathrm{SCN}$ is regulated by different populations that can desynchronize from each other [31, 32]. Indeed a recent study has revealed that splitting of the locomotor activity rhythm may be the consequence of desynchronization of populations between the SCN [33], and another study using a 'forced desynchronization' protocol has shown the presence of two oscillators in the anatomically defined ventrolateral and dorsomedial $\mathrm{SCN}$ subdivisions [34]. Our autoradiography data did not show any obvious evidence of desynchronization between the two SCN subdivisions in each animal (data not shown). However, we cannot categorically exclude that a desynchronization be- tween the two SCN subdivisions has occurred in our animals. Indeed, an increase in the overall variability of Per 1 mRNA was observed in the SCN after 60 days in $L_{d i m}$ (fig. 2). In fact, in the present study Perl expression was only examined in sections obtained from the middle part of the SCN. Another limiting factor in our analysis is represented by the technique we employed (autoradiography followed by a densitometry analysis) that is not suitable to detect small changes in the hybridization signal. Further studies are necessary to test the hypothesis that long-term exposure to $\mathrm{LL}_{\mathrm{dim}}$ may induce desynchronization of a subpopulation of SCN neurons that is responsible for the control of pineal melatonin synthesis.

In conclusion, our data demonstrate that exposure to $\mathrm{LL}_{\mathrm{dim}}$ did not affect the circadian rhythms of Per 1 mRNA in the SCN and locomotor activity, whereas Aa-nat mRNA expression in the pineal gland and the retina of some animals was somewhat affected by exposure to $\mathrm{LL}_{\mathrm{dim}}$ for 60 days. Finally, we found that in all the animals examined melatonin levels showed robust circadian rhythms, although in a few (30\%) animals the serum melatonin rhythm was uncoupled from the circadian rhythms of locomotor activity and Per 1 mRNA expression in the SCN. Finally, it is worthwhile to mention that we observed that pineal Aa-nat mRNA levels showed an altered pattern of expression (i.e., high level during the subjective day or low levels during the subjective night) in a similar percentage of animals that showed altered serum melatonin rhythms.

\section{Acknowledgements}

We thank Dr. H. Okamura for the generous gift of the Period 1 plasmid DNA probe. This work was supported by the NASA Cooperative Agreement NCC 9-58 with the National Space Biomedical Research Institute to G.T.

\section{References}

1 Daan S, Aschoff J: Circadian contribution to survival; in Aschoff J, Daan S, Groos G (eds): Vertebrate Circadian System. Berlin, Springer. 1982, pp 305-321.

2 Czeisler CA, Wright Jr KP: Influence of light on circadian rhythmicity in humans; in Turek FW, Zee PC (eds): Regulation of Sleep and Circadian Rhythms. New York, Dekker, 1999, pp 149-180.
-3 Herzog ED, Tosini G: The mammalian circadian shop. Semin Cell Dev Biol 2001;12:295303.

4 Reppert SM, Weaver DR: Coordination of circadian timing in mammals. Nature 2002;418: 935-941.

-5 Fukuhara C, Dirden JC, Tosini G: Circadian expression of Period1, Period 2 and AA-NAT in the rat pineal under different light conditions. Neurosci Lett 2000;286:167-170.
-6 Sun ZS, Albrecht U, Zhuchenko O, Bailey J, Eichele G, Lee CC: RIGUI, a putative mammalian ortholog of the Drosophila period gene. Cell 1997;90:1003-1011.

7 Tei H, Okamura H, Shigeyoshi Y, Fukuhara C, Ozawa R, Hirose M, Sakaki Y: Circadian oscillation of a mammalian homologue of the Drosophila period gene. Nature 1997;389:512516 
$>8$ Zylka MJ, Shearman LP, Weaver DR, Reppert SM: Three period homologs in mammals: Differential light responses in the suprachiasmatic circadian clock and oscillating transcripts outside of brain. Neuron 1998;20:1103-1110.

$>9$ Klein DC, Coon SL, Roseboom PH, Weller JL, Bernard M, Gastel JA, Zatz M, Iuvone PM, Rodriguez IR, Begay V, Falcon J, Cahill GM, Cassone VM, Baler R: The melatonin rhythmgenerating enzyme: Molecular regulation of serotonin $N$-acetyltransferase in the pineal gland. Recent Prog Horm Res 1997;52:307-358.

10 Sakamoto K, Ishida N: Circadian expression of serotonin $\mathrm{N}$-acetyltransferase mRNA in the rat retina. Neurosci Lett 1998;245:113-116.

- 11 Fukuhara C, Brewer JM, Dirden JC, Bittman EL, Tosini G, Harrington ME: Neuropeptide $Y$ reduces Period 1 and Period 2 mRNA levels in the hamster suprachiasmatic nucleus. Neurosci Lett 2001;314:119-122.

12 Paul C, Fukuhara C, Tosini G, Albers HE: Transduction of light in the suprachiasmatic nucleus: Evidence for two different neurochemical cascades regulating the levels of Per 1 mRNA and Pineal Melatonin. Neuroscience 2003;119:137-144.

13 Fukuhara C, Dirden JC, Tosini G: Regulation of Period1 expression in cultured rat pineal. Neurosignals 2002;11:103-114.

14 Menaker M, Tosini G: The Evolution of vertebrate circadian system; in Honma K-I and Honma S (eds): Circadian Organization and Oscillatory Coupling. Sapporo, Hokkaido University Press, 1996, pp 39-52.

15 Yamazaki S, Numano R, Abe M, Hida A, Takahashi R, Ueda M, Block GD, Sakaki Y, Menaker M, Tei H: Resetting central and peripheral circadian oscillators in transgenic rats. Science 2000;288:682-685.
6 Honma S, Kanematsu N, Katsuno Y, Homna $\mathrm{K}$ : Persistence of circadian oscillation while locomotor activity and plasma melatonin levels became aperiodic under prolonged continuous light in the rat. Neurosci Lett 1996;216: 49-52.

17 Dollins $\mathrm{AB}$, Zhdanova IV, Wurtman RJ Lynch $\mathrm{Hj}$, Deng $\mathrm{MH}$ : Effect of inducing nocturnal serum melatonin concentrations in daytime on sleep, mood, body temperature, and performance. Proc Natl Acad Sci USA 1994; 91:1824-1828.

18 Poulos SG, Borlongan CV: Artificial lighting conditions and melatonin alter motor performance in adult rats. Neurosci Lett 2000;280: 33-36.

19 Tosini G, Menaker M: Circadian rhythm of body temperature in an ectotherm (Iguana iguana). J Biol Rhythms 1995;10:248-255.

20 Sulzman FM, Fuller CA, Moore-Ede MC: Environmental synchronizers of squirrel monkey circadian rhythms. J Appl Physiol 1977;43: 795-800.

21 Erkert HG: Internal desynchronization of the circadian activity and feeding rhythm in an owl monkey (Aotus lemurinus griseimembra): A case study. Chronobiol Int 2000;17:147-153.

22 Ikeda M, Sagara M, Inoue S: Continuous exposure to dim illumination uncouples temporal pattern of sleep, body temperature, locomotion and drinking behavior in rat. Neurosci Lett 2000;279:185-189.

23 Aschoff J, Gerecke U, Weaver R: Desynchronization of human circadian rhythms. Jpn J Physiol 1967; 17:450-457.

24 Lund R: Personality factors and desynchronization of circadian rhythms. Psychosom Med 1974;36:224-228.
25 Koorengevel KM, Beersma DG, Gordijn MC, den Boer JA, van den Hoofdakker RH: Body temperature and mood variation during forced desynchronization in winter depression: A preliminary report. Biol Psychiatry 2000;47:355358.

26 Winget CM, DeRosha CW, Markley CL, Holley DC: A review of human physiological and performance changes associated with desynchronosis of biological rhythms. Aviat Space Environ Med 1984;55:1085-1096.

27 Chen W, Baler R: The rat arylalkylamine Nacetyltransferase E-box: Different use in a master vs. slave oscillator. Mol Brain Res 2000;81: 43-50.

28 Tosini G, Menaker M: Circadian rhythms in cultured mammalian retina. Science 1996;272: 419-421.

29 Klein DC, Moore RY, Reppert SM: Suprachiasmatic Nucleus. The Mind's Clock. New York, Oxford University Press, 1991.

30 Abe M, Herzog ED, Yamazaki S, Straume M, Tei H, Sakaki Y, Menaker M, Block GD: Circadian rhythms in isolated brain regions. J Neurosci 2002;22:350-356.

31 Nakamura W, Honma S, Shirakawa T, Honma $\mathrm{K}$ : Regional pacemakers composed of multiple oscillator neurons in the rat suprachiasmatic nucleus. Eur J Neurosci 2001;14:666-674.

32 Shinohara K, Honma S, Katsuno Y, Abe H, Honma K: Two distinct oscillators in the rat suprachiasmatic nucleus in vitro. Proc Natl Acad Sci USA 1995;92:7396-7340.

33 de la Iglesia H, Meyer J, Carpino A, Schwartz WJ: Antiphase oscillation in the left and right suprachiasmatic nuclei. Science 2000;290: 799-801.

-34 de la Iglesia H, Cambras T, Schwartz WJ, DiezNoguera A: Forced desynchronization of dual oscillators within the rat suprachiasmatic nucleus. Curr Biol 2004; 14:796-800. 